\title{
LA OFTALMÍA PURULENTA EN EL EJÉRCITO ESPAÑOL DURANTE EL SIGLO XIX (1851)
}

\author{
SAMPEDRO A ${ }^{1}$, BARBÓN JJ ${ }^{1}$
}

Fernando Weiler Vice-consultor y Jefe Local en el Hospital Militar de Granada presentó en la Academia de Sanidad Militar de esta Capitanía General en las sesiones de los días 26, 27 y 28 de febrero de 1851 una extensa Memoria sobre la oftalmía purulenta (OP) basándose en los casos atendidos por él en los Hospitales de Palma y Granada. La OP era, con la tisis pulmonar, la enfermedad que mas bajas ocasionaba al Ejército Español en esta época.

El reglamento orgánico del cuerpo de Sanidad Militar de 7 de septiembre de 1846 disponía, en su artículo 15, que se establecieran sesiones mensuales en todos los distritos ó capitanías generales para tratar las cuestiones más relevantes de la medicina castrense. Además, el Director General del cuerpo D. Manuel Codorniu, había establecido que se imprimieran en tomos las memorias más importantes leídas en estas sesiones académicas para darles una difusión general. De ahí surge esta Memoria sobre la oftalmía purulenta que padecen nuestras tropas de Weiler, incluida en el primer tomo de la Biblioteca Médico-castrense española, correspondiente a los meses de mayo y junio de 1851 . Hace un repaso en 9 capítulos sobre la afectación por la oftalmía de otros ejércitos a través de la historia, su posible origen (entonces desconocido), causas, medidas higiénicas para impedir su nacimiento y contener su propagación, síntomas, pronóstico y tratamiento.

La OP es definida por Weiler como: «una inflamación específica, más o menos intensa, sumamente grave, que ataca a la conjuntiva oculo-palpebral, que puede extenderse a las demas partes del ojo, y que va acompañada de diversos sintomas, entre los que sobresalen, un dolor vehemente, un abundante flujo muco-puriforme y la hinchazón de los párpados en el estado agudo, y que cuando llega al crónico produce varias alteraciones en los tejidos afectados, y en particular el desarrollo de granulaciones en la mucosa palpebral».
A lo largo de la historia tuvo otras denominaciones como oftalmía contagiosa, de Egipto, egipcíaca o militar, por haber afectado a los soldados provenientes de las campañas napoleónicas en dicha zona a principios del siglo XIX, que luego extendieron la enfermedad por Europa. Las primeras definiciones son del año 1718 de Próspero Alpino, en su Medi-

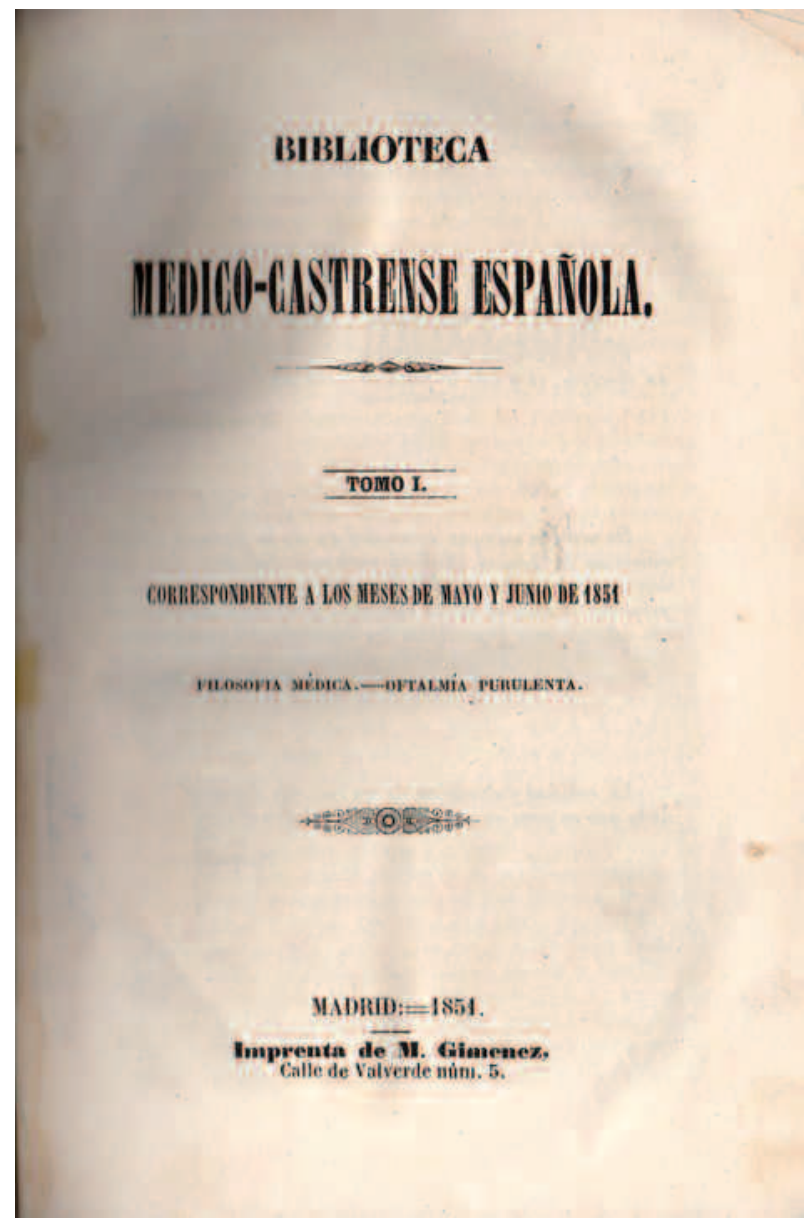

Fig. 1: Primera página del Volumen I de la Biblioteca MEDICO-CASTRENSE ESPAÑOLA.

\footnotetext{
${ }^{1}$ Licenciado en Medicina. Oftalmólogo. Hospital de San Agustín. Avilés. Asturias.

E-mail: ansamlo@hotmail.com
} 
cina Egiptiorum, pero esta enfermedad data de tiempos antiguos, pues en las cruzadas ya resultó afectado San Francisco de Asís precisamente cuando fue a Egipto.

La oftalmía purulenta aparece como «una inflamación, de causa indígena o exótica, desconocida, tal vez atmosférica, seguramente específica, y que por accidentes o circunstancias especiales se convierte en epidémica y contagiosa; que su asiento primitivo es en la mucosa óculo-palpebral, con la propiedad de propagarse a los demas tejidos oculares», sobre todo en situaciones de convivencia masiva como la milicia en las que se daba el hacinamiento, el desaseo de cuerpos, ropas y dormitorios y el uso compartido de otros enseres. Para impedir su nacimiento era imprescindible una esmerada higiene tanto de las personas como de los lugares y, para contener su propagación: «abandonar los lugares infestados, su purificación y la dispersión de los soldados en campamentos y cantones, o su traslación a parages lejanos por medio de largas marchas».

Según nos describe Wharton-Jones en su Tratado práctico de las enfermedades de los ojos, en su edición española de 1862, podríamos distinguir tres grados evolutivos en la OP: en el primero, «la inflamación está en gran parte limitada a la conjuntiva palpebral y al repliegue semilunar; y aunque haya un poco de supuracion puro-mucosa, no hay blenorrea bien manifiesta». En el segundo grado, «la inflamación se extiende a la conjuntiva ocular, que se halla elevada por la exudación y forma un quemosis alrededor de la córnea, con mas ó menos blenorrea». En el tercer grado, «el quemosis es completo, los párpados están enormemente tumefactos, hay un flujo abundante de materia muco-purulenta, y si no están atacadas ya las túnicas propias del globo, corren gran riesgo de ser invadidas». Tan pronto la enfermedad se detiene en el primer grado y se hace crónica, como pasa bruscamente al período mas grave.

Se presentan granulaciones o vegetaciones palpebrales en el segundo periodo que pueden adquirir gran volumen y afectar a la cornea. Rognetta se refiere a estas granulaciones como tracoma según comenta Weiler (usada por los antiguos para referirse a asperidad y granulaciones en general).

El tratamiento de la OP incluye en la fase aguda: antiflogísticos directos como «la flebotomía, la arteriotomía, y las evacuaciones locales por medio de sanguijuelas», y antiflogísticos indirectos o hipostenizantes como: «baños generales, las vento- sas, los pediluvios, vejigatorios, sedales, fonticulos, moxas, cáusticos y demás revulsivos; añadiendo el régimen alimenticio, los purgantes minorativos y los verdaderos hipostenizantes, como la digital, el nitro, los calomelanos, la ipecacuana, el tártaro estibiado, los sudoríficos, narcóticos, etc.» La tumefacción y la quemosis conjuntival se podían tratar, además, con escisiones, desbridamiento y cauterización. De los medios utilizados, para el autor «sólo tres merecen la reputación de la que gozan: tales son el colirio egipcio, el tanino y el nitrato de plata».

En los estadios crónicos debían destruirse las granulaciones «con la escisión con pinzas, tijeras ó bisturí, si son fungosas, pediculadas ó voluminosas; y si menores o aglomeradas, con escarificaciones y cauterizantes, como el sulfato de cobre, y el nitrato de plata, solo o con el de potasa, fundidos juntos».

Tabla I. Movimiento mensual de altas y bajas

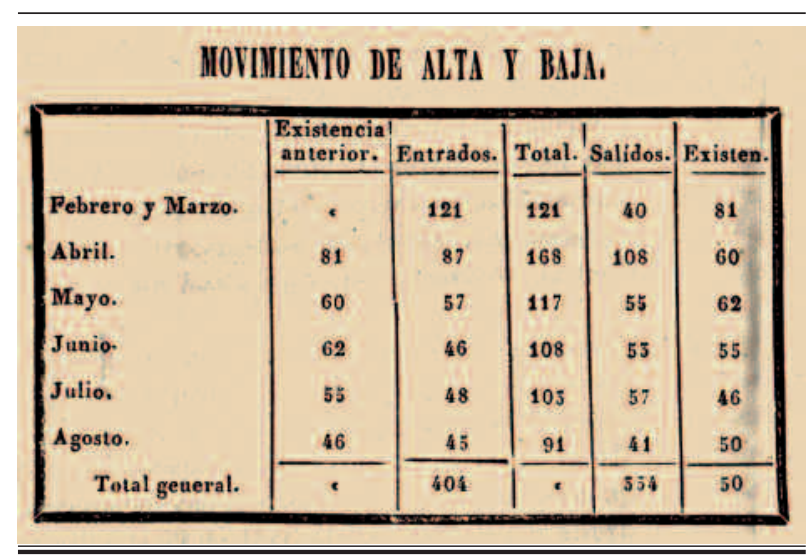

Tabla II. Tiempos empleados para curación de la oftalmía

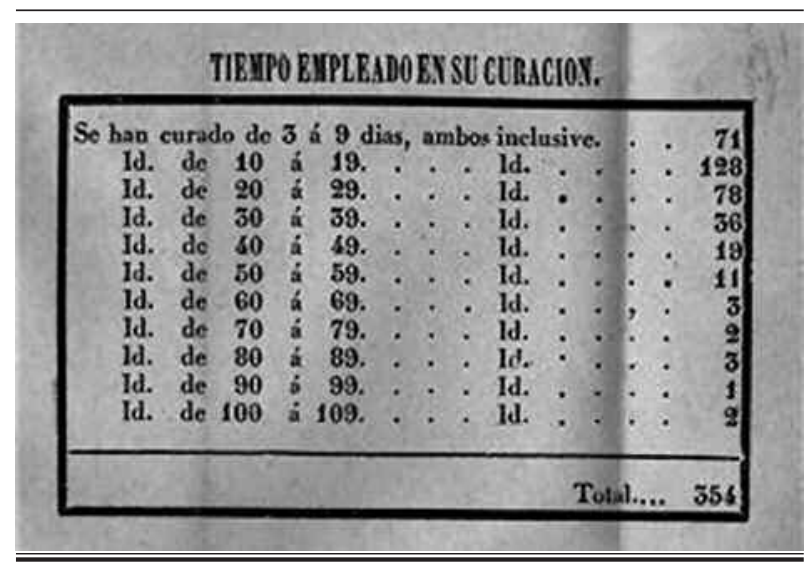


Weiler presenta unas estadísticas de 404 enfermos tratados en el Hospital Militar de Granada en un periodo de seis meses, de los que ocho fueron declarados inútiles para el Servicio Militar, uno con ceguera bilateral y otros cuatro con pérdidas severas unilaterales. El autor incluye unos cuadros muy ilustrativos sobre los movimientos de altas y bajas de los soldados de los Regimientos de África asistidos con oftalmía purulenta y el tiempo empleado para su curación (tablas I y II).

Esta OP se corresponde con la conjuntivitis tracomatosa, contagiosa a través de las secreciones oculares, vehiculadas a través de toallas, dedos, pañuelos, etc., sobre todo cuando existía un contacto estrecho entre personas, como sucedía en escuelas, asilos y cuarteles con higiene deficiente. El tracoma evoluciona con frecuentes exacerbaciones y recaídas y las complicaciones más importantes son el pannus y la ulceración corneal dejando como secuelas la triquiasis, el entropión, ectropión, xerosis y opacidades de la córnea. Este proceso podía también ser confundido, en su forma más grave, con una conjuntivitis gonocócica del adulto, ya que cursa con quemosis tumefacción palpebral y secreción purulenta. En ambas conjuntivitis infecciosas se desconocían los gérmenes causantes, Chlamydia trachomatis y Neisseria gonorrhoeae y en aquel tiempo se trataba igual la conjuntivitis gonocócica que la oftalmía egipciaca en su tercer grado.

\section{BIBLIOGRAFÍA}

1. Wharton-Jones T. Tratado práctico de las enfermedades de los ojos. Madrid: Ed. Moya; 1862.

2. Biblioteca Médico-Castrense Española. Madrid: Sanidad Militar; 1851.

3. Haab O. Atlas-manual de las enfermedades externas del ojo. Madrid: Librería Académica; 1908. 